Office for National Statistics data using Kaplan-Meier survival graphs, Pivot tables, multivariate analysis (Cox proportional hazard model) and log rank testing using SPSS v19 and Excel 2010.

Mesothelioma incidence, diluted by a rural hinterland, rose over 10 years from 2 to 16 per million compared with HSE data for overall UK incidence (2006-8) of 62.3 per million (male) and 11.6 per million (female). Annual mesothelioma deaths as a proportion of all deaths rose over 10 years from 0.02 to $0.17 \% .87 \%$ cases were male, mean age of presentation was 72 years (range 34-95 years), and overall median and $95 \%$ Confidence Interval (CI) survival of 8 (5.8-10.2) months.

Median (CI) survival was 11 (8.6-13.4) months for patients $<75$ years age, greater than for $\geq 75$ years of 3 (0.7-5.3) months $(p=0.005)$. There was a significantly greater survival of patients with performance status (PS) 0 or 1 at 11 (8.2-13.8) months compared to PS 2.3 or 4 at 2, (9-3.1; $p=0.0000002)$ months.

Multivariate analysis, using a Cox proportional hazards regression model showed epithelioid histology subtype predicted longer survival compared to sarcomatoid (Hazard ratio 0.11 (CI 0.02-0.52; $\mathrm{p}=0.005)$; see Fig.1).

Survival analysis using a log rank test showed no significant difference between median (CI) survival of men 8 (6.0-10.0) months and women $5(0-11.9 ; \mathrm{p}=0.7)$ months. Other variables not significantly affecting survival were side of tumour $(p=0.9)$, and diagnosing hospital $(p=0.07)$.

Extrapleural pneumonectomy (EPP) was performed on 10 patients (5 male, PS 0-1, age 34-76 years). Survival for patients receiving EPP was 20.0 (3.3-36.7) months, significantly greater than 7.0 (4.2-9.5) months for all patients $(p=0.023)$. No statistically significant survival benefit for EPP was seen compared with survival of 49 patients matched for age and PS ( $p=0.182)$; these findings were confirmed by multivariate analysis.

We conclude that mesothelioma represents an increasing burden and that patient factors, not treatment, govern survival.
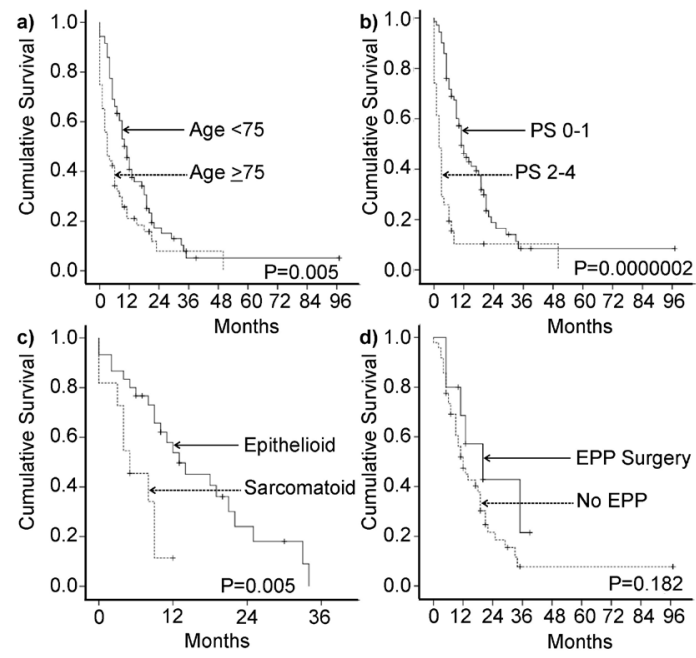

Abstract P198 Figure 1

\section{P199 MERSEYSIDE \& CHESHIRE CANCER NETWORK (MCCN) AUDIT: SURVIVAL ANALYSIS OF LUNG CANCER PATIENTS WITH A PERFORMANCE STATUS OF 3 (PS3) BASED UPON THE REASON FOR THEIR POOR PERFORMANCE STATUS AND WHETHER OR NOT THEY HAD A TISSUE DIAGNOSIS}

doi:10.1136/thoraxjnl-2012-202678.260

IP Owen, '2J Hendry. 'Merseyside \& Cheshire Cancer Network, Bromborough, UK; ${ }^{2}$ St Helens \& Knowsley Teaching Hospitals NHS Trust, Prescot, UK
Introduction and Objectives Lung Cancer patients with a PS3 often fall on the boundary between fitness for active oncological treatments and "best supportive care". Some patients with PS3 are deteriorating rapidly because of advanced cancer symptoms and have a very poor prognosis. Others have stable chronic health problems that influence PS.

Aims

- To determine the percentage of patients with PS3 who fall into these subcategories and compare 12 month survival

- To ask whether having a tissue diagnosis in patients with PS3 improves outcome.

Assigning a PS3 to a patient may influence whether or not a tissue diagnosis is pursued. In some cases this may be because the patient has severe cardiorespiratory disease and a biopsy is unsafe. In other cases, the multidisciplinary team may decide that the patient is not fit to have active oncological treatment so pursuing a biopsy would not alter management, and might even be unethical because of the risk incurred.

Methods All lung cancer units in MCCN were asked to complete a questionnaire for 50 consecutive Lung Cancer patients with PS3 (most were diagnosed in 2010), which was then compared with death data from the North West Cancer Intelligence Service.

Results Survival is generally greater in patients who are given a PS3 because they are stable due to chronic illness (34.5\% were alive after 12 months) and poorer in those deteriorating due to advanced cancer symptoms (4.1\%) or a combination of the two (13.1\%).

In neither patients with PS3 because of ill health, nor patients with PS3 because of advanced cancer symptoms was there a clear survival advantage for patients who had a tissue diagnosis of cancer as opposed to a clinical and radiological diagnosis.

Conclusions Based on this data, there is no explicit survival advantage in pursuing tissue diagnosis in patients with PS3. In our network, those patients with PS3 who are fit for active oncological treatment received it even when it was considered inappropriate to pursue histology. In the future, should targeted therapies become available that can be tolerated in patients with PS3, the case for pursuit of histology will be stronger.

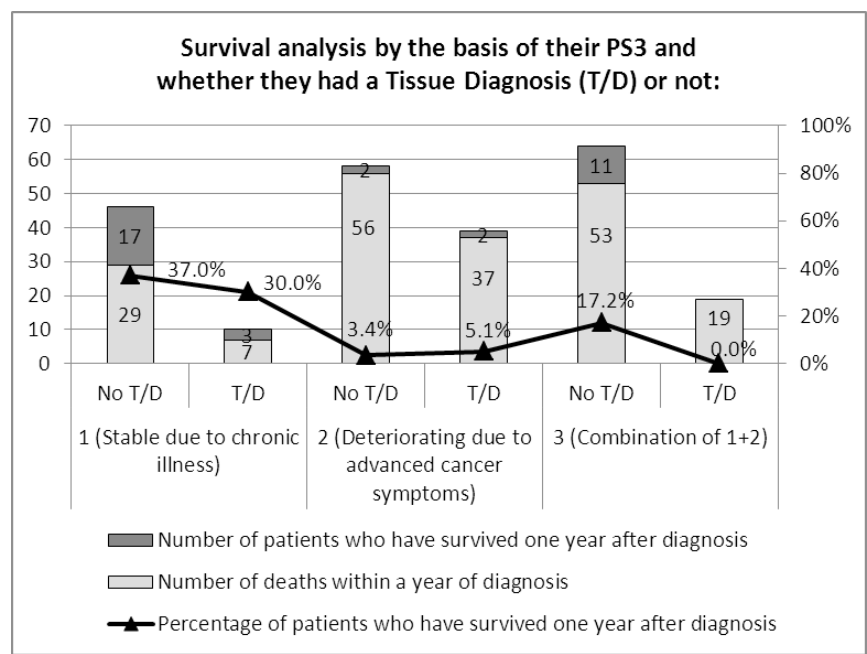

Abstract P199 Figure 1

\section{P200 SIX YEAR EXPERIENCE WITH RIGID BRONCHOSCOPY: COMPLICATIONS, INDICATIONS AND CHANGING REFERRAL PATTERNS}

doi:10.1136/thoraxjnl-2012-202678.261

JL Bacon, SK Leaver, BP Madden. St George's Hospital, London, United Kingdom

Introduction and Objectives The objective of this study was to determine the indications and outcomes of the last 500 referrals for 